\title{
Residue Induced Product Failures-Microanalysis
}

\author{
Paul M. Verghese ${ }^{1}$, Noah Budiansky ${ }^{1}$, Paul Ledwith ${ }^{1}$, and David Bauer ${ }^{1}$ \\ ${ }^{1}$ Exponent, Inc., Natick, MA, USA
}

Foreign residues can result in a number of different types of product failures. For example, residues can facilitate corrosion that leads to mechanical or electrical failures; they can result in unacceptable changes in visual appearance, such as staining or smudges; and residues can interfere with mechanical movement, such as switch actuation or connector engagement. Residues introduced by manufacturing processes or in service life are often unpredictable but are capable of resulting in major problems for product manufacturers and consumers. In failure analysis investigations, it is often important to analyze residues to help identify them and their possible origins in order to mitigate these problems.

Oftentimes, a pragmatic approach involves the use of multiple analytical techniques, applied in a sequential fashion from relatively simple methods like visual examination and optical microscopy, to more complex but readily available methods, like Fourier transform infrared spectroscopy (FTIR) and scanning electron microscopy (SEM) with energy dispersive X-ray spectroscopy (EDS). Sometimes enough useful information can be gleaned from these initial analyses that more advanced analytical methods are not needed to complete the investigation. However, depending on the needs of the investigation and the complexity of a particular residue, more advanced analytical techniques might be necessary in order to develop a more complete understanding, such as mass spectroscopic methods, advanced electron or ion beam microscopy, and highly sensitive surface chemical analysis.

During regular usage, portable electronics are often exposed to liquids that can eventually result in performance degradation and/or device failure. In many cases corrosion of portable device components provides a visual indicator of foreign contaminant exposure, although the presence of corrosion on a particular component need not implicate that component as the cause of device failure. For example, analysis of a mobile phone that was exposed to a liquid contaminant resulted in corrosion of a galvanized retention screw (Figure 1). White and brown surface residues were present on the screw head. Elemental analysis of these surface residues (Figure 2) showed evidence of zinc, iron, and oxygen, which suggests the presence of oxide corrosion products when considered together with the residue's visible appearance. In addition, carbon, potassium, chlorine, phosphorous, and calcium were observed in the residue areas. FTIR was conducted to complement the elemental analysis by providing chemical functional group information, which can help to further identify organic and inorganic compounds. In this case, FTIR detected fructose, suggesting ingress of a beverage or other similar product, as shown in Figure 3. Further elemental and FTIR analysis of suspected liquid products confirmed that the device had likely been exposed to a sports beverage.

In this work we present several foreign residue case studies of varying complexity, covering a variety of residues, failure modes, and residue analysis methodologies that were used during the failure investigations. 

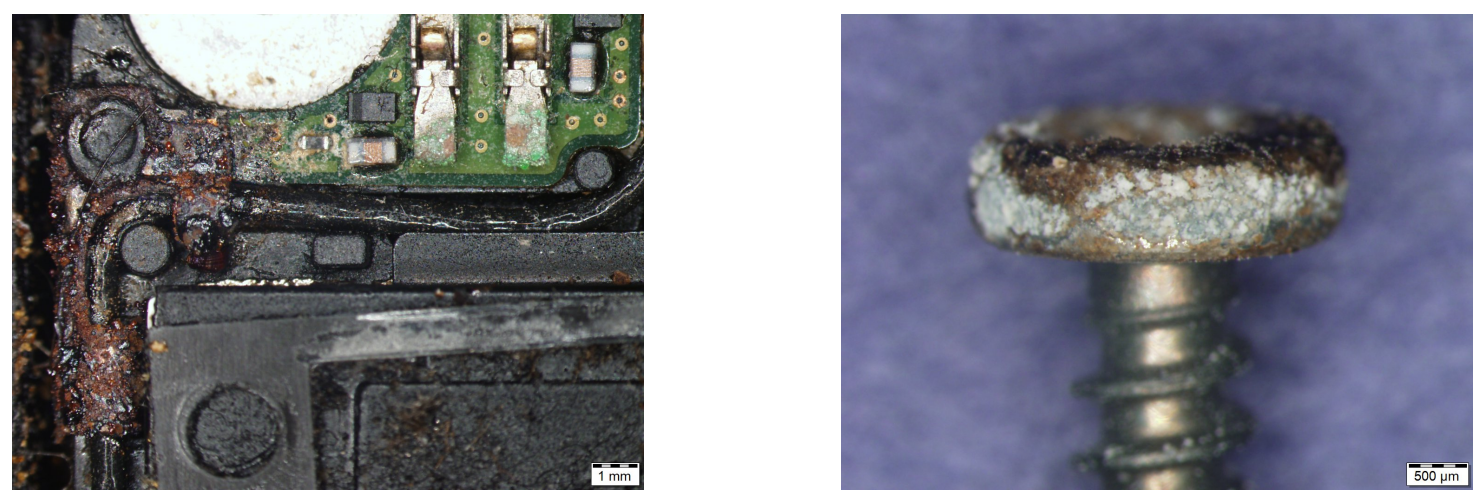

Figure 1. Residues and corrosion on mobile phone components; (A) printed circuit board assembly, (B) screw fastener
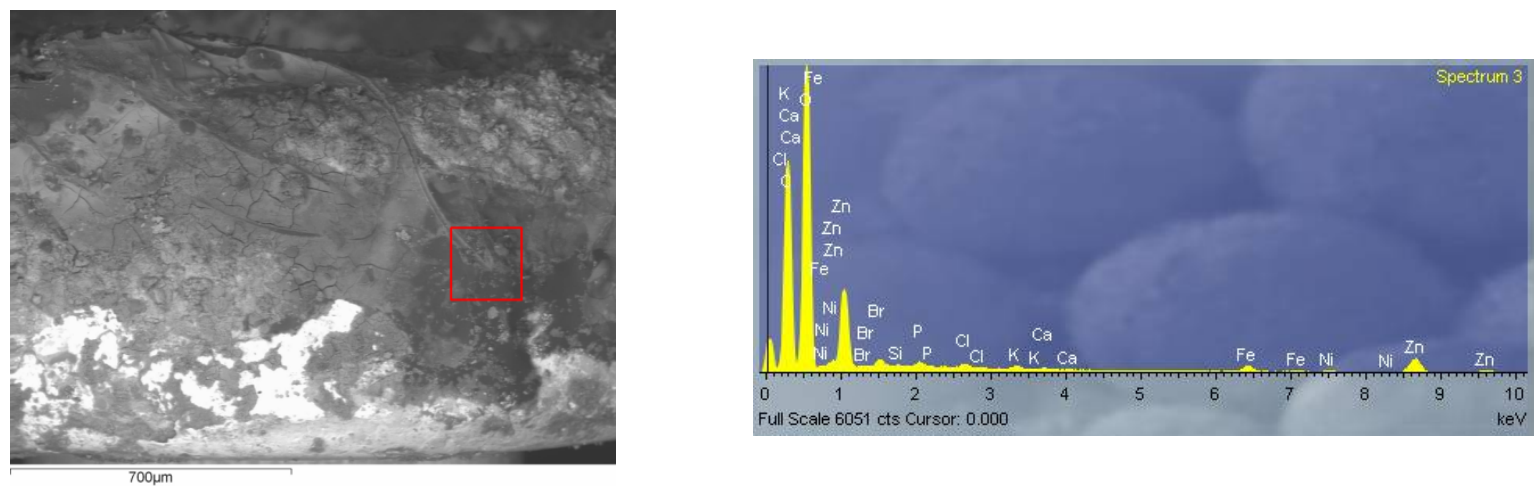

Figure 2. SEM image and EDS spectrum of residue on screw fastener

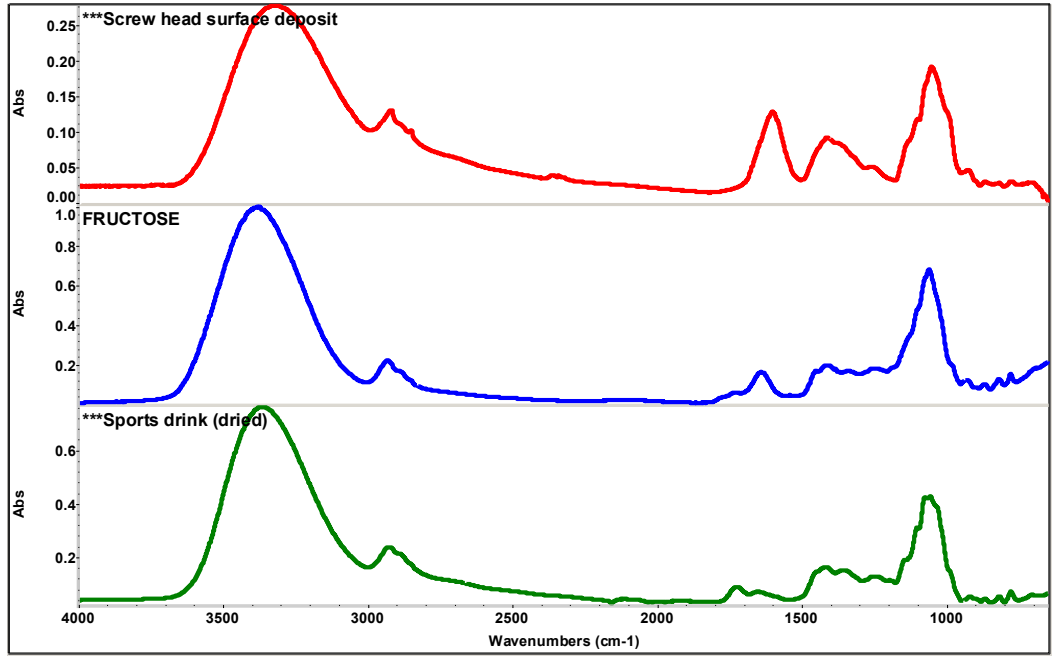

Figure 3. FTIR spectrum of an area of dried residue located on the head of the examined screw; the bottom two spectra are library reference spectra shown for comparison. 